Check for updates

Cite this: RSC Adv., 2019, 9, 29246

\title{
Microwave magnetoimpedance and ferromagnetic resonance in $\mathrm{Pr}_{0.6} \mathrm{Sr}_{0.4} \mathrm{MnO}_{3}$
}

\begin{abstract}
A. Chanda (iD) and R. Mahendiran (D) *
We report the magnetic field dependence of electrical impedance (magnetoimpedance) of a ferromagnetic $\mathrm{Pr}_{0.6} \mathrm{Sr}_{0.4} \mathrm{MnO}_{3}$ sample carrying alternating current (ac) of frequency $f=1 \mathrm{MHz}$ to $3 \mathrm{GHz}$ measured using an impedance analyzer and broad band ferromagnetic resonance ( $f=2$ to $18 \mathrm{GHz}$ ) measured using a coplanar wave guide based spectrometer. Ac magnetoresistance is much larger than dc magnetoresistance and its sign at low magnetic fields changes from negative to positive with increasing frequency of the ac current. The field dependence of ac magnetoresistance shows a peak around $H_{\mathrm{dc}}=0$ for low frequencies but a double peak feature emerges at $H_{\mathrm{dc}}= \pm H_{\mathrm{p}}$ at higher frequencies and it shifts to higher magnetic field as the frequency of ac current increases. The field derivative of microwave power absorption measured by the broad band spectrometer shows features of ferromagnetic resonance and the resonance field increases with increasing frequency of microwave radiation following Kittel's equation for ferromagnetic resonance. A close correlation is found between the ferromagnetic resonance line shape and the positive peak in the ac magnetoresistance, which suggests the possibility of electrical detection of ferromagnetic resonance using high frequency current injected into a conducting magnetic sample.
\end{abstract}

Received 27th August 2019

Accepted 12th September 2019

DOI: $10.1039 / c 9 r a 06786 h$

rsc.li/rsc-advances magnetoresistance with alternating currents has potential for practical applications compared to the dc magnetoresistance if the low-field sensitivity can be further improved.

Since hopping of the $\mathrm{e}_{\mathrm{g}}$ electron between $\mathrm{Mn}^{3+}\left(\mathrm{t}_{2 \mathrm{~g}}^{3} \mathrm{e}_{\mathrm{g}}^{1}\right)$ and $\mathrm{Mn}^{4+}\left(\mathrm{t}_{2 \mathrm{~g}}^{3} \mathrm{e}_{\mathrm{g}}^{0}\right)$ via the intervening oxygen anion is responsible for electrical conduction in manganites, it is also of scientific curiosity to know how the resistivity and magnetoresistance are affected if the $e_{g}$ electron is forced to oscillate at $\mathrm{MHz}$ and $\mathrm{GHz}$ frequencies. M. Dominguez et al. ${ }^{9}$ reported $70 \%$ magnetoresistance for 600 Oe near its Curie temperature $\left(T_{\mathrm{C}}\right)$ in $\mathrm{Nd}_{0.7} \mathrm{Sr}_{0.3^{-}}$ $\mathrm{MnO}_{3}$ thin film and $\mathrm{V}$. Srinuvasu et al. ${ }^{\mathbf{1 0}}$ found $80 \%$ magnetoresistance for a field of 600 Oe near $T_{\mathrm{C}}$ in $\mathrm{La}_{0.7} \mathrm{Ba}_{0.3^{-}}$ $\mathrm{MnO}_{3}$ powder when these samples placed inside a microwave resonant cavity were irradiated with electromagnetic fields in microwave frequency range $(f=10 \mathrm{GHz})$. Microwave magnetoresistance was estimated from changes in microwave reflectivity of the cavity in absence and in presence of a dc magnetic field. In contrast to these reports, here, we report microwave magnetotransport in $\mathrm{Pr}_{0.6} \mathrm{Sr}_{0.4} \mathrm{MnO}_{3}$ by passing alternating current (ac) of frequency $f$ through the sample and measuring its electrical impedance in presence of an external dc magnetic field.

$\mathrm{Pr}_{0.6} \mathrm{Sr}_{0.4} \mathrm{MnO}_{3}$ is ferromagnetic at room temperature $\left(T_{\mathrm{C}}=\right.$ $305 \mathrm{~K})$ but its magnetization shows a step-like change around $T_{\mathrm{S}}$ $=89 \mathrm{~K}\left(\ll T_{\mathrm{C}}\right)$, which is apparently triggered by orthorhombic (Pnma space group) to monoclinic (I/2a space group) structural transition. ${ }^{\mathbf{1 1 2}}$ Interestingly, this compound exhibits normal and inverse magnetocaloric effects at $T_{\mathrm{C}}$ and $T_{\mathrm{S}}$, respectively. ${ }^{13} \mathrm{~A}$ close correlation was also found between the magnetic entropy change, magnetothermopower and magnetization. While dc 
resistivity showed a very weak anomaly at $T_{\mathrm{S}}$ in a single crystalline sample, ${ }^{\mathbf{1 4}}$ it was not detectable in the polycrystalline sample. However, ac resistance $(1 \mathrm{MHz}<f<5 \mathrm{MHz})$ showed a pronounced anomaly at $T_{\mathrm{S}}$. It is of our interest to understand the behavior of magnetoresistance at frequencies higher than 5 MHz. Here, we extend the magnetotransport measurement in $\mathrm{Pr}_{0.6} \mathrm{Sr}_{0.4} \mathrm{MnO}_{3}$ over a wide frequency range $(f=1 \mathrm{MHz}$ to $3 \mathrm{GHz})$ in response to radio frequency current flowing through the sample. In addition, we also report microwave absorption for multiple frequencies $(f=2$ to $10 \mathrm{GHz})$ of microwave electromagnetic field. Our results indicate that high frequency magnetoimpedance shows features of ferromagnetic resonance (FMR). Although FMR and electron spin resonance (ESR) in Kand Na-doped $\mathrm{Pr}_{0.6} \mathrm{Sr}_{0.4} \mathrm{MnO}_{3}$ (ref. 15) as well as other manganites ${ }^{\mathbf{1 6 - 1 8}}$ have been studied using conventional MW cavity resonators, FMR excited by MW current flowing through the sample has not been investigated. Here, we demonstrate a broadband detection of FMR in $\mathrm{Pr}_{0.6} \mathrm{Sr}_{0.4} \mathrm{MnO}_{3}$ by electrical means using an impedance analyzer. The main advantage of our magnetoimpedance (MI) method is the broad operational frequency range unlike the conventional MW cavity resonator based technique which usually operates at a fixed frequency (around $9.8 \mathrm{GHz}$ ) in X-band. Because the MI technique probes spin dynamics, it can be exploited to obtain Gilbert's damping parameter, which is not possible with a single frequency cavity resonator FMR technique.

\section{Experimental details}

Polycrystalline sample of $\mathrm{Pr}_{0.6} \mathrm{Sr}_{0.4} \mathrm{MnO}_{3}$ was prepared by conventional solid state reaction method. It crystallizes in single phase with orthorhombic structure (space group Pnma) at room temperature. ${ }^{19}$ Magnetization was measured using a vibrating sample magnetometer (VSM) and four probe electrical resistivity was measured in a Physical Property Measuring System (PPMS). The sample was cut into a rectangular plate of dimensions $5 \mathrm{~mm} \times 3 \mathrm{~mm} \times 2 \mathrm{~mm}$ for both dc resistivity and magnetoimpedance measurement. A radio frequency impedance analyzer (Agilent E4991A) was used to measure the resistive $(R)$ and reactive $(X)$ components of the complex impedance, $Z(f)=R(f)+\mathrm{i} X(f)$ of the sample by employing the radio frequency (rf) current-voltage method. As shown in the Fig. 1(a), the sample placed on an Agilent test probe stage is attached to the signal line at one end and the other end of the sample is connected to the ground plate using silver paint. To avoid electrical contact between the sample surface and the ground plate, a layer of kapton tape was inserted between them. The rf current flows through the sample from the signal line to the ground plane. The test probe stage is placed at the center of an electromagnet. We define the angle between the direction of $\mathrm{rf}$ current flow and that of the dc magnetic field $\left(H_{\mathrm{dc}}\right)$ produced by the electromagnet by $\theta$. The $R$ and $X$ at selected frequencies were measured while sweeping $H_{\mathrm{dc}}$ for $\theta=0^{\circ}, 30^{\circ}, 60^{\circ}$ and $90^{\circ}$. We define dc magnetoresistance as $\operatorname{MR}_{\mathrm{dc}}=[\rho(H)-\rho(H=0)] / \rho(H=$ $0) \times 100 \%$, where, $\rho$ is the dc electrical resistivity of the sample. AC magnetoresistance is defined as $\mathrm{MR}_{\mathrm{ac}}=[R(H, f)-R(H=0$, $f)] / R(H=0, f) \times 100 \%$ for a frequency $f$ of alternating current

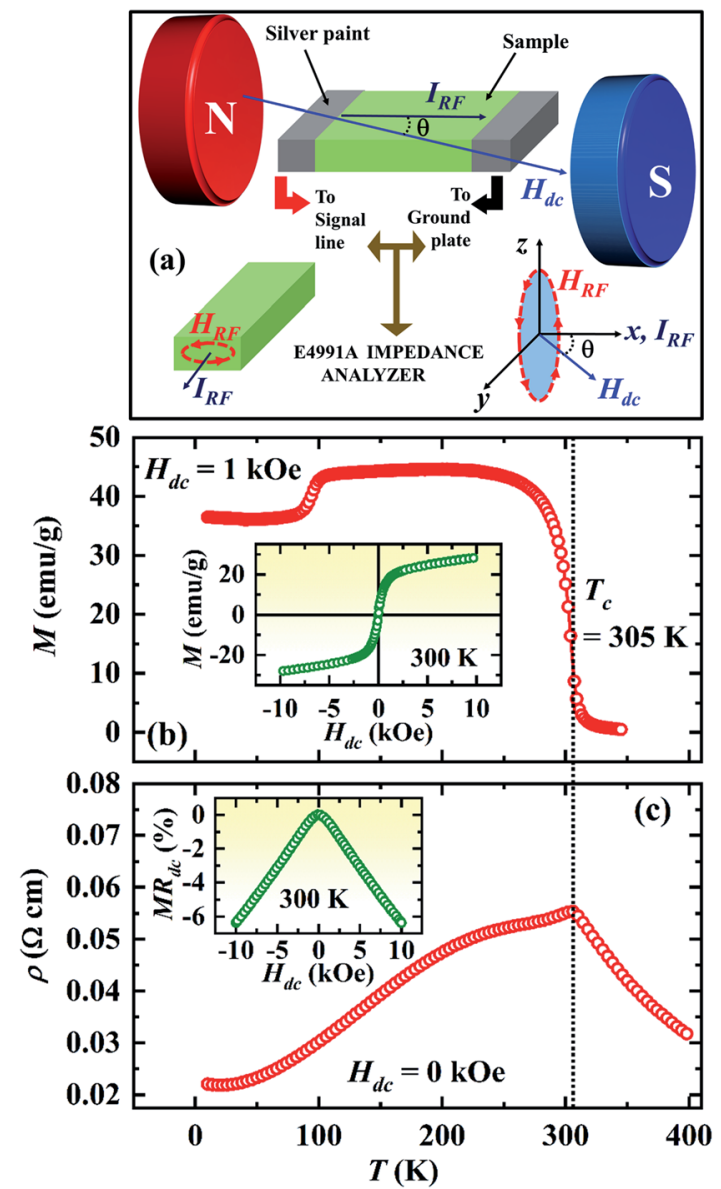

Fig. 1 (a) A schematic illustration of the broadband magnetoimpedance experimental configuration. (b) Temperature dependence of magnetization, $M(T)$ of $\mathrm{Pr}_{0.6} \mathrm{Sr}_{0.4} \mathrm{MnO}_{3}$ in a magnetic field of $\mathrm{H}_{\mathrm{dc}}=1$ $\mathrm{kOe}$, inset shows $M(H)$ isotherm at $300 \mathrm{~K}(\mathrm{c})$ temperature dependence of four-probe dc resistivity of $\mathrm{Pr}_{0.6} \mathrm{Sr}_{0.4} \mathrm{MnO}_{3}$, inset shows the $\mathrm{dc}$ magnetoresistance, $\mathrm{MR}_{\mathrm{dc}}$ at $300 \mathrm{~K}$.

and the magnetoreactance is defined as $\mathrm{MX}=[X(H, f)-X(H=$ $0, f)] / X(H=0, f) \times 100 \%$. We also define magnetoimpedance as, $\mathrm{MZ}=[Z(H, f)-Z(H=0, f)] / Z(H=0, f) \times 100 \%$, where, $Z=\left(R^{2}+\right.$ $\left.X^{2}\right)^{1 / 2}$. The magnetic field dependence of the field derivative of microwave power absorption $(\mathrm{d} P / \mathrm{d} H)$ was measured using a commercial broadband ferromagnetic resonance spectrometer (NanOsc Phase-FMR from Quantum Design Inc.) that makes use of coplanar waveguide method. The PPMS was used to provide an in-plane dc magnetic field $\left(H_{\mathrm{dc}}\right)$ in such a way that the rf magnetic field $\left(H_{\mathrm{rf}}\right)$ generated in the waveguide is perpendicular to $H_{\mathrm{dc}}$ (see Fig. 4(a)). The dc magnetic field was modulated using a pair of Helmholtz coils, which provided low amplitude (1 Oe) and low frequency $(440 \mathrm{~Hz}$ ) ac magnetic field $\left(H_{\mathrm{ac}}\right)$. Because of the field modulation and the lock-in technique used, microwave power absorption was recorded as the field derivative $(\mathrm{d} P / \mathrm{d} H)$ as like in conventional ESR spectrometers.

\section{Results}

The main panel of Fig. 1(b) shows $M(T)$ of $\operatorname{Pr}_{0.6} \mathrm{Sr}_{0.4} \mathrm{MnO}_{3}$ sample in a dc magnetic field of $H_{\mathrm{dc}}=1 \mathrm{kOe}$ while cooling from 
$T=350 \mathrm{~K}$. The ferromagnetic Curie temperature $\left(T_{\mathrm{C}}\right)$ determined from the minimum of $\mathrm{d} M / \mathrm{d} T$ curve is $305 \mathrm{~K}$. The inset of Fig. 1(b) shows the magnetic field dependence of magnetization at $T=300 \mathrm{~K}$ which confirms the soft ferromagnetic nature of the sample. The temperature dependence of dc resistivity $\rho(T)$ (see the main panel of Fig. 1(c)) measured in zero external magnetic field shows a change from thermally activated behavior in the paramagnetic state to metallic behavior below $T_{\mathrm{C}}$ with a peak occurring at $T_{\mathrm{C}}$. The dc magnetoresistance $\left(\mathrm{MR}_{\mathrm{dc}}\right)$ at $T=300 \mathrm{~K}$ (inset of Fig. 1(c)) shows a single peak at $H_{\mathrm{dc}}$ $=0$ with a negative sign and increases in magnitude. The absolute value of $\mathrm{MR}_{\mathrm{dc}}$ is $6 \%$ at $H_{\mathrm{dc}}= \pm 10 \mathrm{kOe}$.

Fig. 2(a) shows the magnetic field dependence of the ac magnetoresistance $\left(\mathrm{MR}_{\mathrm{ac}}\right)$ at room temperature for different frequencies of the alternating current $(f=1 \mathrm{MHz}$ to $3 \mathrm{GHz})$. The dc magnetic field $\left(H_{\mathrm{dc}}\right)$ produced by the electromagnet is parallel to the direction of rf current $\left(I_{\mathrm{rf}}\right)$ passing through the sample $\left(\theta=0^{\circ}\right) . \mathrm{MR}_{\mathrm{ac}}$ is negative at $f=1 \mathrm{MHz}$ with an absolute value of $2 \%$ at $H_{\mathrm{dc}}=5 \mathrm{kOe}$ and shows a single peak at $H_{\mathrm{dc}}=0$. As the frequency of the alternating current increases, the absolute value of $\mathrm{MR}_{\mathrm{ac}}$ increases up to a certain frequency $(\sim 500 \mathrm{MHz})$ but decreases for still higher frequencies. Above $f=$ $500 \mathrm{MHz}$, the single peak at $H_{\mathrm{dc}}=0$ splits into two symmetrical peaks at $H_{\mathrm{dc}}= \pm H_{\mathrm{p}}$ on either sides of the zero field and both these peaks migrate towards higher magnetic fields as $f$ increases further. The sample shows a positive ac MR in the field range $-H_{\mathrm{p}} \leq H_{\mathrm{dc}} \leq+H_{\mathrm{p}}$ for $f>500 \mathrm{MHz}$.

Fig. 2(b) shows the magnetic field dependence of the magnetoreactance (MX). MX also shows double peaks at $H_{\mathrm{dc}}=$ $\pm H_{\mathrm{q}}\left(H_{\mathrm{q}}>H_{\mathrm{p}}\right)$ above $500 \mathrm{MHz}$ which also move towards higher field with increasing frequency. The double peaks in $\mathrm{MX}$ at $\pm H_{\mathrm{q}}$, unlike in $\mathrm{MR}_{\mathrm{ac}}$, fade above $f=1 \mathrm{GHz}$. In addition to the double peaks, MX also shows a single peak centered at origin $\left(H_{\mathrm{dc}}=0\right)$ above $2 \mathrm{GHz}$ culminating in double dips between $H_{\mathrm{dc}}=0$ and $\pm H_{\mathrm{q}}$. The field dependence of magnetoimpedance (MZ) shown in Fig. 2(c) is almost identical to that of MX.

The main panel of Fig. 3(a) displays the frequency dependence of $\mathrm{MR}_{\mathrm{ac}}$ at the highest field for $\theta=0^{\circ}$. The absolute value of negative ac MR increases with increasing frequency and becomes maximum around $500 \mathrm{MHz}(\sim 22 \%)$ but decreases towards zero with further increasing frequency. On the other hand, the value of positive ac MR at $H_{\mathrm{dc}}= \pm H_{\mathrm{p}}$ increases gradually with increasing frequency and reaches $\sim 23 \%$ (see the inset of Fig. 3(a)) for $f=3 \mathrm{GHz}$. It is noteworthy that the value of $\mathrm{MR}_{\mathrm{dc}}$ at $5 \mathrm{kOe}$ is only $3 \%$ which is almost 7 times lower than the value of $\mathrm{MR}_{\mathrm{ac}}$ at the same field for $f=500 \mathrm{MHz}$.

We have also studied how the observed anomalous features vary if the angle between radio frequency (rf) current direction
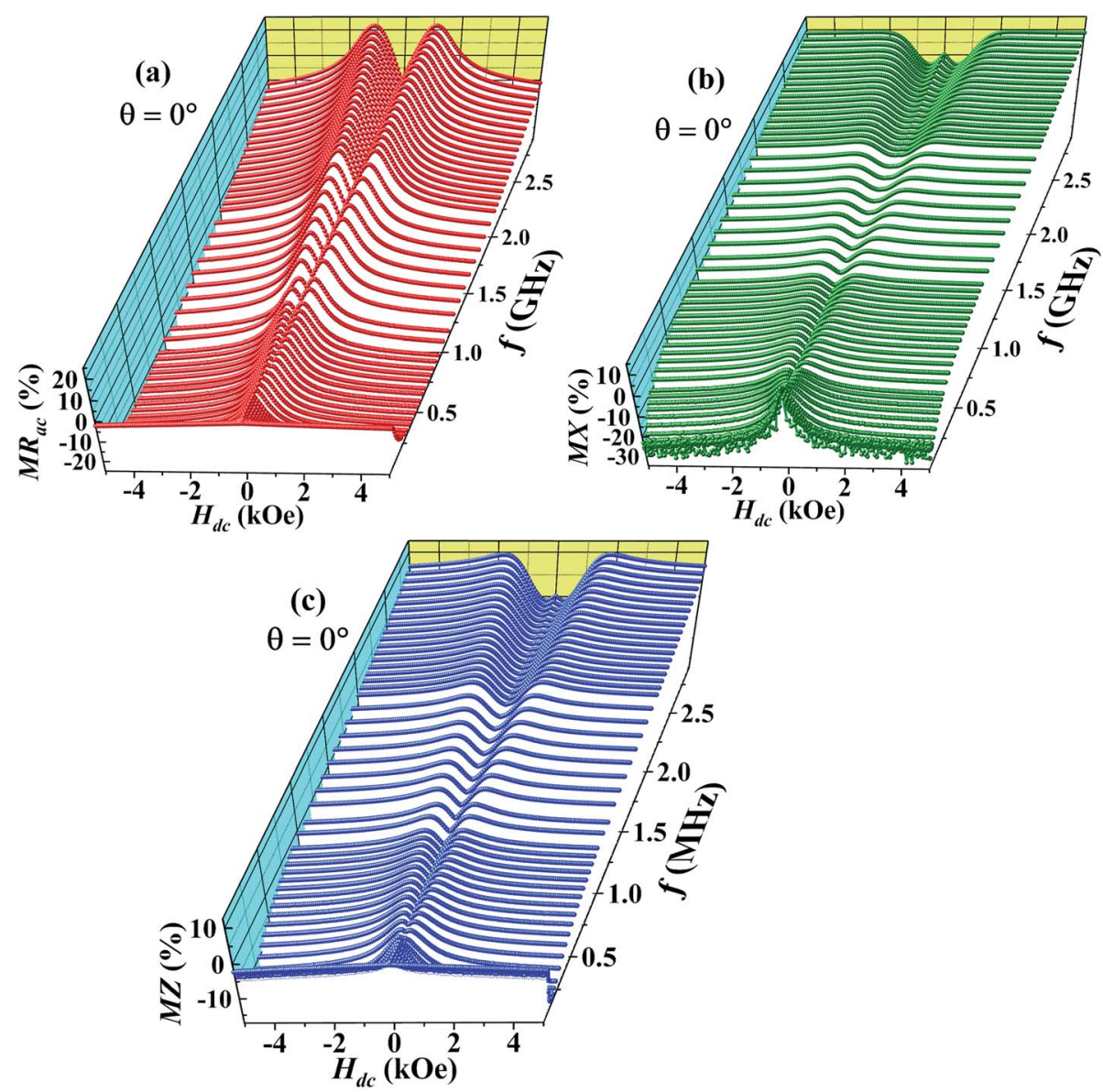

Fig. 2 Magnetic field dependence of (a) ac magnetoresistance $\left(M R_{a c}\right)$ (b) magnetoreactance (MX) and (c) magnetoimpedance (MZ) as a percentage change at $T=300 \mathrm{~K}$ for $\theta=0^{\circ}$ for different frequencies of the ac current between $f=1 \mathrm{MHz}$ and $3 \mathrm{GHz}$. 

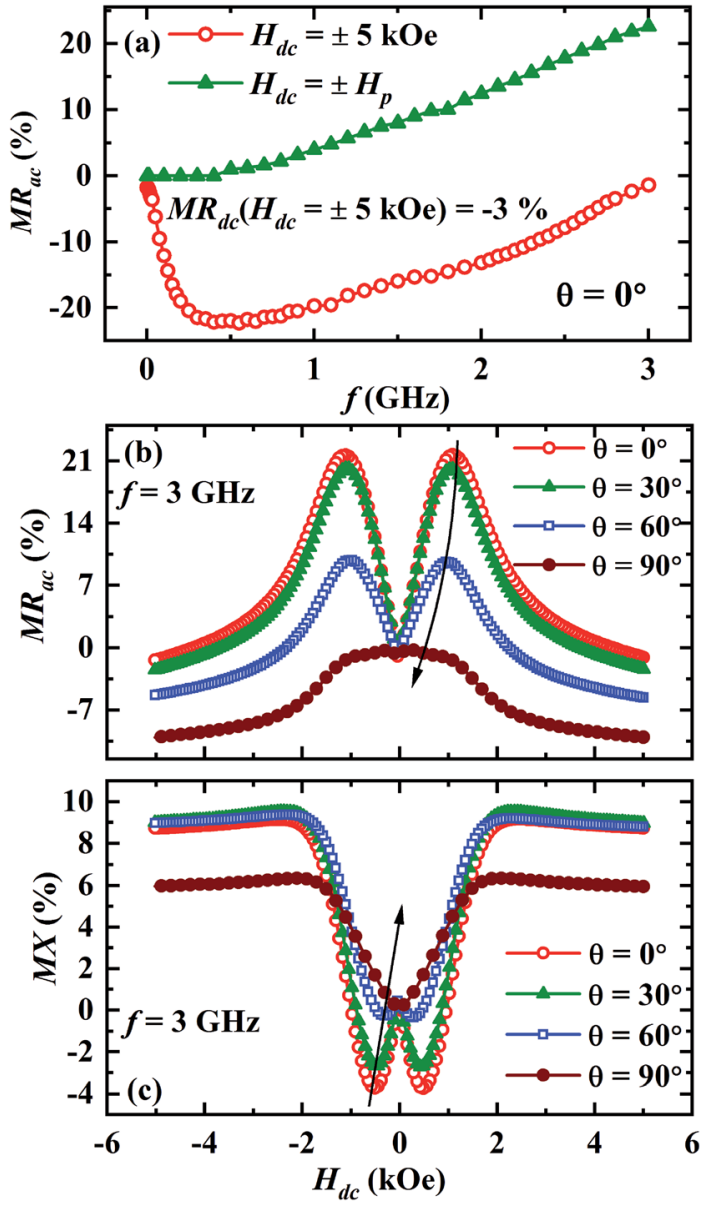

Fig. 3 (a) The ac MR as a function of frequency at room temperature for $\theta=0^{\circ}$, magnetic field dependence of (b) $M R_{a c}$ and (c) $M X$ for $\theta=0^{\circ}$, $30^{\circ}, 60^{\circ}$ and $90^{\circ}$ at a fixed frequency $f=3 \mathrm{GHz}$.

and the direction of dc magnetic field $(\theta)$ is changed. $\mathrm{MR}_{\mathrm{ac}}$ and MX for $\theta=0^{\circ}, 30^{\circ}, 60^{\circ}$ and $90^{\circ}$ are depicted in the Fig. $3(\mathrm{~b})$ and (c), respectively for $f=3 \mathrm{GHz}$. It is clear that the intensity of the double peaks at $H_{\mathrm{dc}}= \pm H_{\mathrm{p}}$ in $\mathrm{MR}_{\mathrm{ac}}$ as well as the double dips at $H_{\mathrm{dc}}= \pm H_{\mathrm{q}}$ in MX decreases with increasing $\theta$. Moreover, the double peak (dip) positions in $\mathrm{MR}_{\mathrm{ac}}(\mathrm{MX})$ also shift to lower fields as $\theta$ increases and they disappear completely for $\theta=90^{\circ}$.

Fig. 4(b) shows the magnetic field dependence of microwave power absorption recorded as the field derivative $(\mathrm{d} P / \mathrm{d} H)$ for different frequencies of the microwave magnetic field between $f$ $=2-10 \mathrm{GHz}$ at room temperature. The microwave absorption signal for each frequency exhibits a magnetic resonance feature a dip at a higher field followed by a peak at a lower field when the dc field is reduced from a high value to zero. The signal for 2 GHz shows a peak around $H_{\mathrm{dc}}=160$ Oe. The peak in $\mathrm{d} P / \mathrm{d} H$ shifts towards higher $H_{\mathrm{dc}}$ as the frequency of the microwave electromagnetic field increases similar to the behavior of ac MR.

To understand a possible link between the anomalous behaviors of $\mathrm{MR}_{\mathrm{ac}}$ and $\mathrm{MX}$, we plot the $\mathrm{MR}_{\mathrm{ac}}$ (left scale) and the MX (right scale) together in a single graph for $f=3 \mathrm{GHz}$ in Fig. 5(a). A careful look reveals that the peak in $\mathrm{MR}_{\mathrm{ac}}$ isotherm almost coincides with the point of inflection in MX isotherm
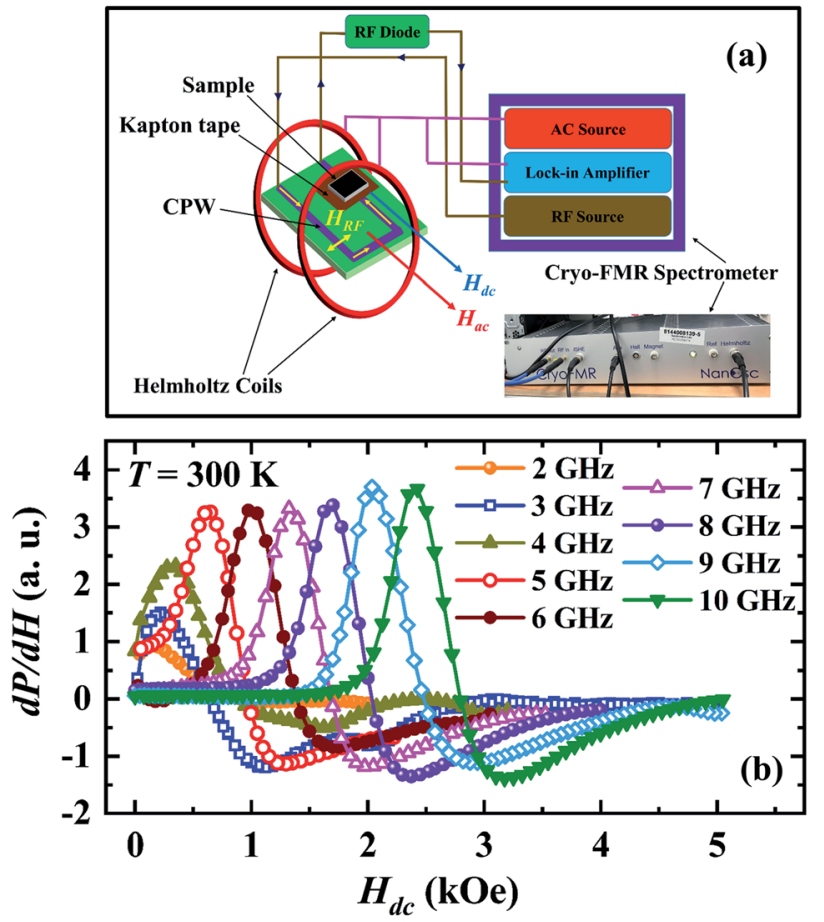

Fig. 4 (a) A schematic representation of the broadband FMR setup, (b) magnetic field dependence of the field derivative of the microwave power absorption $(\mathrm{d} P / \mathrm{d} H)$ at $T=300 \mathrm{~K}$ at selected fixed frequencies of the microwave magnetic field ( $f=2$ to $10 \mathrm{GHz}$ ).

but MX is nearly independent of $H_{\mathrm{dc}}$ above 2 kOe unlike that of the $\mathrm{MR}_{\mathrm{ac}}$. It is clear from the Fig. 5 (b) that the peak in $\mathrm{MR}_{\mathrm{ac}}$ (left $y$-axis) coincides with the peak in the field derivative of MX
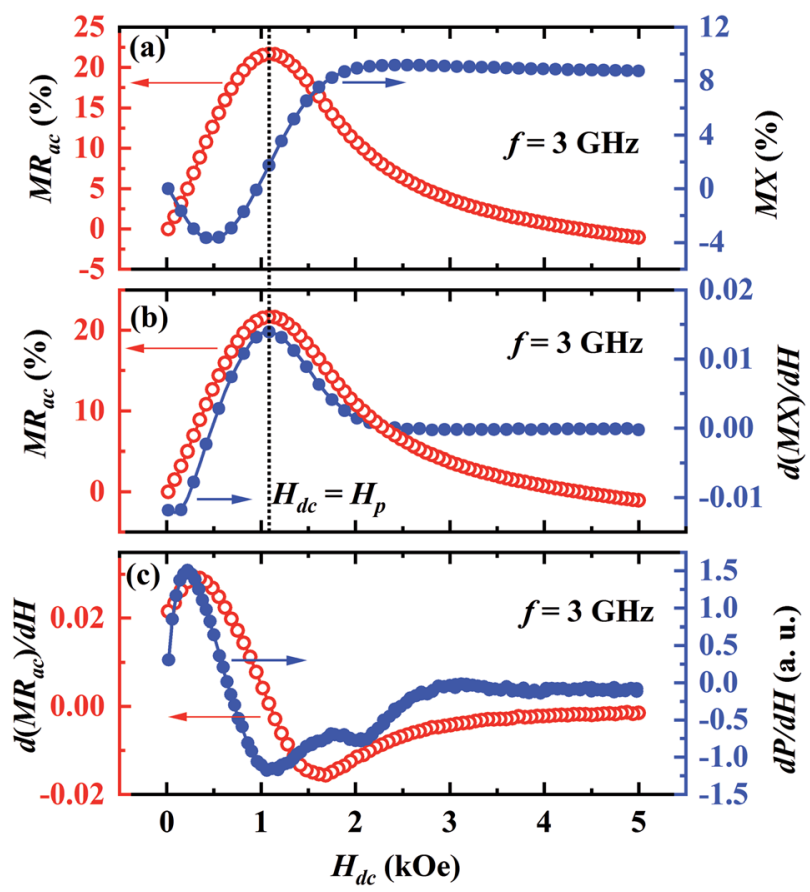

Fig. 5 (a) $M R_{a c}$ on the left scale and $M X$ on the right scale, (b) $M R_{a c}$ on the left $y$ scale and first derivative of the $M X(d(M X) / d H)$ on the right scale, (c) first derivative of the $M R_{a c}\left(d\left(M R_{a c}\right) / d H\right)$ on the left $y$ scale and $(\mathrm{d} P / \mathrm{d} H)$ on the right scale for $f=3 \mathrm{GHz}$ at $T=300 \mathrm{~K}$. 
(right $y$-axis). In Fig. 5(c), we plot the field derivative of the $\mathrm{MR}_{\mathrm{ac}}$ on the left $y$-axis and $\mathrm{d} P / \mathrm{d} H$ on the right $y$-axis for $f=3 \mathrm{GHz}$. It is evident that the peak positions of $\mathrm{d}\left(\mathrm{MR}_{\mathrm{ac}}\right) / \mathrm{d} H$ and $\mathrm{d} P / \mathrm{d} H$ closely match with each other. However, the linewidth of $\mathrm{d}\left(\mathrm{MR}_{\mathrm{ac}}\right) / \mathrm{d} H$ is higher than that of $\mathrm{d} P / \mathrm{d} H$.

\section{Discussion}

We summarize important observations from the above results as follows: (1) $\mathrm{Pr}_{0.6} \mathrm{Sr}_{0.4} \mathrm{MnO}_{3}$ is a room temperature ferromagnet with $T_{\mathrm{C}}=305 \mathrm{~K}$. (2) The ac magnetoresistance $\mathrm{MR}_{\mathrm{ac}}$ is negative and shows a peak at $H_{\mathrm{dc}}=0$ for $f \leq 500 \mathrm{MHz}$ but exhibits positive double peaks at $H_{\mathrm{dc}}= \pm H_{p}$ for higher frequencies. (3) The absolute value of negative ac MR at $H_{\mathrm{dc}}=5$ kOe increases with frequency and becomes maximum $(\sim 22 \%)$ at $f=500 \mathrm{MHz}$ which is significantly higher than the value of dc MR ( $-3 \%)$ at the same field. (4) The value of positive $M_{\mathrm{ac}}$ at $H_{\mathrm{dc}}= \pm H_{\mathrm{p}}$ increases with frequency and becomes $\sim+23 \%$ for $f$ $=3 \mathrm{GHz}$. (5) As $\theta$ increases from resonant $\left(0^{\circ}\right)$ to non-resonant $\left(90^{\circ}\right)$ configuration, the double peak at $H_{\mathrm{dc}}= \pm H_{\mathrm{p}}$ in the $\mathrm{MR}_{\mathrm{ac}}$ shifts towards the origin as well as the value of $\mathrm{MR}_{\mathrm{ac}}$ at $H_{\mathrm{dc}}=$ $\pm H_{\mathrm{p}}$ decreases considerably. (6) The magnetic field corresponding to the peak in $\mathrm{MR}_{\mathrm{ac}}$ isotherm coincides with the point of inflection of MX isotherm. (7) The $\mathrm{d} P / \mathrm{d} H$ shows a Lorentzian line shape and the maximum in $\mathrm{d} P / \mathrm{d} H$ shifts towards higher $H_{\mathrm{dc}}$ with increasing frequency. (8) The peak positions in $\mathrm{d}\left(\mathrm{MR}_{\mathrm{ac}}\right) / \mathrm{d} H$ and $\mathrm{d} P / \mathrm{d} H$ closely match with each other.

Let us first shed some light on the origin of such anomalous behavior of the ac magnetotransport in this sample. The application of a dc electric field to the sample forces the mobile $\mathrm{e}_{\mathrm{g}}$ electron to hop between $\mathrm{Mn}^{3+}$ and $\mathrm{Mn}^{4+}$ ions in the background of immobile $t_{2 g}^{3}$ core electrons. The hopping is dependent on the relative angle between $t_{2 g}$ spins of $\mathrm{Mn}$ ions. While dc or low frequency ac current flows uniformly throughout the volume of the sample, high frequency current tends to flow only in thin surface layer of thickness " $\delta$ " due to skin effect. The "skin depth" $\delta$ decreases with increasing angular frequency $(\omega$ $=2 \pi f$ ) of the ac current and dependent on dc resistivity $(\rho)$ and transverse magnetic permeability $\left(\mu_{\mathrm{t}}\right)$ of the sample through the expression, $\delta=\left(\frac{2 \rho}{\omega \mu_{0} \mu_{\mathrm{t}}}\right)^{1 / 2}$, where, $\mu_{0}$ is the free space permeability. Instead of resistance for dc current, we consider impedance for alternating current. The electrical impedance of a rectangular slab with thickness $2 t$ and infinite width is given by, ${ }^{20}$

$$
Z=R_{\mathrm{dc}} k t \operatorname{coth}(k t)
$$

where, $k=(1+i) / \delta$ is the wave vector and $R_{\mathrm{dc}}$ is the dc resistance of the sample. Using $\rho=55 \times 10^{-5} \Omega \mathrm{m}$ at $300 \mathrm{~K}$ and taking $\mu_{\mathrm{t}}=$ 1 corresponding to the non-magnetic limit, the value of $\delta$ in our sample at $f=3 \mathrm{GHz}$ is $220 \mu \mathrm{m}$ which is almost 10 times smaller than the sample thickness $(2 \mathrm{~mm})$. When the skin effect is very strong, i.e., $\delta \ll 2 t, k t \gg 1$ and hence, coth $(k t) \rightarrow 1$. In that case, the expression for surface impedance of the sample $Z$ becomes, ${ }^{20}$

$$
Z=R_{\mathrm{dc}} t(1+i)\left(\frac{\omega \mu_{0} \mu_{\mathrm{t}}}{2 \rho}\right)^{\frac{1}{2}}
$$

Besides the skin effect, the flow of radio frequency (rf) current in the sample generates a transverse circular rf magnetic field $\left(H_{\mathrm{rf}}\right)$ which interacts with the magnetization of the sample and hence, affects the transverse component of the magnetic permeability $\left(\mu_{\mathrm{t}}\right)$. The transverse permeability is a complex quantity and given by $\mu_{\mathrm{t}}=\mu_{\mathrm{t}}^{\prime}-i \mu_{\mathrm{t}}^{\prime \prime}$ where, $\mu_{\mathrm{t}}^{\prime}$ is the in-phase component (magnetization is in-phase with the rf magnetic field) and $\mu_{t}^{\prime \prime}$ is the out-of-phase component (magnetization is not in-phase with the rf magnetic field).

Hence, the complex surface impedance is given by, ${ }^{21}$

$$
Z=\frac{1}{2 \sqrt{2 t}} \sqrt{\omega \rho \mu_{0}}\left(\sqrt{\mu_{\mathrm{R}}}+i \sqrt{\mu_{\mathrm{X}}}\right)
$$

where,

$$
\mu_{\mathrm{R}}=\sqrt{\mu_{\mathrm{t}}{ }^{2}+\mu_{\mathrm{t}}^{\prime \prime 2}}+\mu_{\mathrm{t}}^{\prime \prime}
$$

and $\mu_{\mathrm{x}}=\sqrt{\mu_{\mathrm{t}}{ }^{2}+\mu_{\mathrm{t}}^{\prime \prime 2}}-\mu_{\mathrm{t}}^{\prime \prime}$.

Hence, the real (R) and imaginary (X) parts of the complex impedance are $\quad \mathrm{R}=\frac{1}{2 \sqrt{2 t}} \sqrt{\omega \rho \mu_{0}\left(\sqrt{\mu_{\mathrm{t}}^{\prime 2}+\mu_{\mathrm{t}}^{\prime \prime 2}}+\mu_{\mathrm{t}}^{\prime \prime}\right)}$ and $\mathrm{X}=\frac{1}{2 \sqrt{2 t}} \sqrt{\omega \rho \mu_{0}\left(\sqrt{\mu_{\mathrm{t}}^{\prime 2}+\mu_{\mathrm{t}}^{\prime \prime 2}}-\mu_{\mathrm{t}}^{\prime \prime}\right)}$, respectively. Since $\mu_{\mathrm{t}}$ is affected by external dc magnetic field, it influences the field dependence of both $\mathrm{R}$ and $\mathrm{X}$. So, unlike dc magnetoresistance, ac magnetoresistance in the high frequency regime is dominated mostly by magnetization dynamics of the sample rather than changes in scattering rate of the conducting electrons.

When the applied dc magnetic field $\left(H_{\mathrm{dc}}\right)$ is smaller than the saturation field, $\mu_{\mathrm{t}}$ of the sample depends on the relative alignment of the magnetic easy axis and the direction of $H_{\mathrm{dc}}$. If $H_{\mathrm{dc}}$ is parallel to the easy axis, $\mu_{\mathrm{t}}$ decreases monotonically from its maximum value in zero field to nonmagnetic limit $\left(\mu_{\mathrm{t}}=1\right)$ with increasing strength of the dc magnetic field. However, if $H_{\mathrm{dc}}$ is perpendicular to the magnetic easy axis, magnetization of the sample rotates but does not switch towards $H_{\mathrm{dc}}$ unless $H_{\mathrm{dc}}$ exceeds the anisotropy field $H_{\mathrm{k}}$. The transverse permeability is expected to diverge at $H=H_{\mathrm{k}}$ for an ideal system but usually shows a peak at $H_{\mathrm{k}}$ in non-ideal case, ${ }^{20-22}$ Since, $R=\frac{1}{2 \sqrt{2 t}} \sqrt{\omega \rho \mu_{0}\left(\sqrt{\mu_{\mathrm{t}}^{\prime 2}+\mu_{\mathrm{t}}^{\prime \prime 2}}+\mu_{\mathrm{t}}^{\prime \prime}\right)}$, the ac resistance or surface resistance increases with $H_{\mathrm{dc}}$ and shows a peak at $H_{\mathrm{dc}}=$ $\pm H_{\mathrm{p}}$, where $H_{\mathrm{p}}$ is close to $H_{\mathrm{k}}$, which explains the appearance of double peaks in $\mathrm{MR}_{\mathrm{ac}}$ of our sample in the frequency range $f=$ 500-1000 MHz for the orthogonal configuration of $H_{\mathrm{dc}}$ and $H_{\mathrm{rf}}$ $\left(\theta=0^{\circ}\right)$. The disappearance of double peak behaviour in $\mathrm{MR}_{\mathrm{ac}}$ for $\theta=90^{\circ}$ is expected because $\mu_{\mathrm{t}}$ will decrease monotonically with $H_{\mathrm{dc}}$ for the parallel configuration of $H_{\mathrm{dc}}$ and $H_{\mathrm{rf}}$. The shift of R-peak at $H_{\mathrm{dc}}= \pm H_{\mathrm{p}}$ with frequency is small in the frequency regime $f=500-1000 \mathrm{MHz}$ which could be due to dispersion in the anisotropy field (only few tens of Oe). ${ }^{23}$ However, gyromagnetic effect, i.e., precession of magnetization about the direction of dc magnetic field dominates at frequencies higher than $500 \mathrm{MHz}$. When the frequency of magnetization precession matches with that of the rf magnetic field, steady state 
precession of magnetization, i.e., ferromagnetic resonance (FMR) occurs in the sample. The FMR frequency $f_{\text {res }}$ for a thin plate like sample increases with the frequency following the Kittel's expression when $H_{\mathrm{dc}}$ is perpendicular to $H_{\mathrm{rf}},{ }^{24}$

$$
f_{\mathrm{res}}=\frac{\gamma}{2 \pi} \sqrt{\left[\left(H_{\mathrm{dc}}+H_{\mathrm{k}}\right)\left(H_{\mathrm{dc}}+H_{\mathrm{k}}+M_{\mathrm{eff}}\right)\right]}
$$

where, $H_{\mathrm{k}}$ is the transverse anisotropy field, $M_{\mathrm{eff}}$ is the effective magnetization whose value is close to saturation magnetization $\left(M_{\mathrm{S}}\right)$ and $\gamma$ is the gyromagnetic ratio, $\gamma=g \mu_{\mathrm{B}} / \hbar$, where " $g$ " is the "Lande' $g$-factor".

Britel et al., showed that a close link exists between the magnetoimpedance measured by electrical method and the FMR recorded using a microwave electron spin resonance (ESR) spectrometer. ${ }^{25}$ In an ESR spectrometer, a sample placed inside a microwave (MW) resonant cavity is irradiated with electromagnetic field in microwave range, usually 9-10 GHz. The magnetic component of electromagnetic field impinging on the sample inside MW cavity is transverse to the dc magnetic field produced externally by an electromagnet. Similarly, the rf/MW magnetic field generated by rf/MW current in the sample is also transverse to the dc magnetic field in our magnetoimpedance measurement when $\theta=0^{\circ}$. The sample absorbs maximum power from the MW electromagnetic field during FMR. The power absorption $(P)$ in the sample per unit volume is related to the out-of-phase component of transverse permeability through the expression

$$
P=\frac{1}{2} \chi_{\mathrm{t}}^{\prime \prime} \omega h_{\mathrm{rf}}^{2}
$$

where, $h_{\mathrm{rf}}$ is the amplitude of the alternating magnetic field $\left(H_{\mathrm{rf}}(t)=H_{0}+h_{\mathrm{rf}} \cos \omega t\right), \chi_{\mathrm{t}}^{\prime \prime}\left(=\mu_{\mathrm{t}}^{\prime \prime}\right)$ is the out-of-phase component of transverse susceptibility. $\mu_{\mathrm{t}}^{\prime \prime}$ goes through a maximum at the resonance field $\left(H_{\mathrm{res}}\right)$ and hence, $P\left(H_{\mathrm{dc}}\right)$ also shows maximum at $H_{\mathrm{dc}}=H_{\text {res }}$ Since $R=\frac{1}{2 \sqrt{2 t}} \sqrt{\omega \rho \mu_{0}\left(\sqrt{\mu_{\mathrm{t}}^{\prime 2}+\mu_{\mathrm{t}}^{\prime \prime 2}}+\mu_{\mathrm{t}}^{\prime \prime}\right)}$, the appearance of peak in $\mathrm{MR}_{\mathrm{ac}}$ at $H_{\mathrm{dc}}= \pm H_{\mathrm{p}}$ can be considered as a consequence of the maximum power absorption by the sample due to FMR. Similar behavior was reported in magnetic field dependence of impedance $(Z)$ for $\mathrm{Fe}-\mathrm{Co}-\mathrm{Si}-\mathrm{B}$ amorphous ribbons ${ }^{26}$ where the field corresponding to the peak in real component of $Z$ shifts rapidly to higher fields with increasing frequency. D. de Cos et al. ${ }^{27}$ also found a close correlation between $\mathrm{d} R_{\mathrm{ac}} / \mathrm{d} H$ obtained from magnetoimpedance and $\mathrm{d} P / \mathrm{d} H$ obtained from microwave power absorption in cavity resonator.

The magnetic field dependence of power absorption, $P\left(H_{\mathrm{dc}}\right)$ usually shows a Lorentzian line shape described by the expression, ${ }^{28}$

$$
P\left(H_{\mathrm{dc}}\right)=P_{\max } \frac{\left(\frac{\Delta H}{2}\right)^{2}}{\left(H_{\mathrm{dc}}-H_{\mathrm{res}}\right)^{2}+\left(\frac{\Delta H}{2}\right)^{2}}
$$

where, $P_{\max }$ is the absorbed power at the resonance field $\left(H_{\mathrm{dc}}=\right.$ $\left.H_{\text {res }}\right)$ and $\Delta H$ is the full line width at half the peak power (FWHM). Since the magnetic field dependence of $R$ reflects the behavior of $P\left(H_{\mathrm{dc}}\right)$, the field dependence of the high frequency
$\mathrm{MR}_{\mathrm{ac}}$ can also be described by the Lorentzian function. We have fitted the $\mathrm{MR}_{\mathrm{ac}}$ line shapes with the following equation, ${ }^{29}$

$$
\begin{aligned}
\mathrm{MR}_{\mathrm{ac}}\left(H_{\mathrm{dc}}\right)= & R_{\mathrm{sym}} \frac{\left(\frac{\Delta H}{2}\right)^{2}}{\left(H_{\mathrm{dc}}-H_{\mathrm{res}}\right)^{2}+\left(\frac{\Delta H}{2}\right)^{2}} \\
& +R_{\mathrm{asym}} \frac{\frac{\Delta H}{2}\left(H_{\mathrm{dc}}-H_{\mathrm{res}}\right)}{\left(H_{\mathrm{dc}}-H_{\mathrm{res}}\right)^{2}+\left(\frac{\Delta H}{2}\right)^{2}}+R_{0}
\end{aligned}
$$

where, $R_{\text {sym }}$ and $R_{\text {asym }}$ are the coefficients of symmetric and antisymmetric Lorentzian functions, $H_{\text {res }}$ and $\Delta H$ are the resonance field and linewidth, respectively for the $\mathrm{MR}_{\mathrm{ac}}$ line shape and $R_{0}$ is a constant offset parameter. Eqn (7) is composed of a symmetric and an antisymmetric Lorentzian function which is generally used for the electrical detection of FMR. ${ }^{29,30}$ The symmetry of FMR line shape depends on the relative phase between microwave electric and magnetic field components. ${ }^{29}$ The symmetric line shape accounts for the contribution of $\mathrm{rf}$ current which is in-phase with the rf magnetization and the antisymmetric line shape accounts for the out-of-phase contribution. ${ }^{31}$ Thus, the resultant line shape is a linear combination of symmetric and antisymmetric contributions which leads to the asymmetric nature of the FMR line shape. ${ }^{29}$

Fig. 6(a) displays the fitting of the $\mathrm{MR}_{\mathrm{ac}}$ line shape with the eqn (7) for a few selected frequencies between $f=1$ and $3 \mathrm{GHz}$.
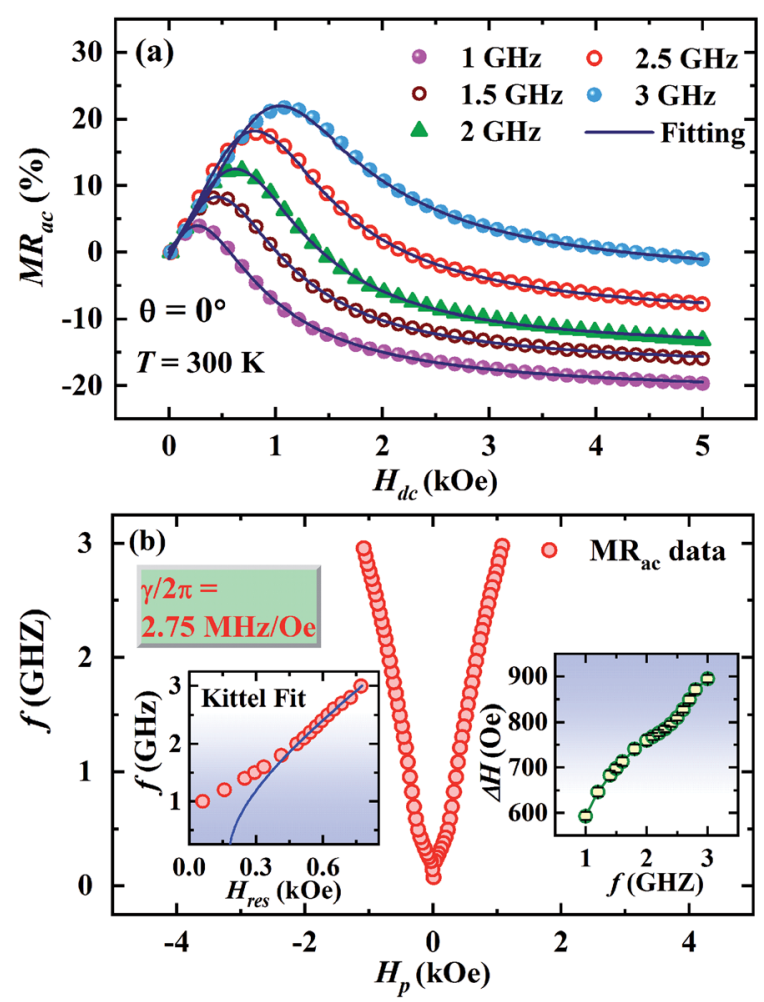

Fig. 6 (a) Fitting of $M R_{a c}$ line shapes for $\theta=0^{\circ}$. (b) Main panel: a plot of frequency $(f)$ as a function of the dc magnetic field $\left(H_{p}\right)$ at which the ac MR shows a peak, left inset shows Kittel-fit to the plot of $f$ vs. resonance field $\left(H_{\text {res }}\right)$ extracted from the $M R_{a c}$ data and right inset shows frequency dependence of linewidth $(\Delta H)$. 
The main panel of Fig. 6(b) shows $H_{\mathrm{dc}}$ corresponding to the peak in $\mathrm{MR}_{\mathrm{ac}}$ on $x$-scale and the corresponding frequency on $y$ scale which clearly indicates the evolution of double peaks and their rapid upshifting in $H_{\mathrm{dc}}$ with increasing frequency of ac current. The plot $f v s$. resonance field $\left(H_{\text {res }}\right)$ above $1 \mathrm{GHz}$ obtained from the fitting of the $\mathrm{MR}_{\mathrm{ac}}$ line shape are shown in the left inset of Fig. 6(b). Fitting of the $f v s$. $H_{\text {res }}$ curve with the Kittel's equation for FMR described by eqn (4) yields $\gamma / 2 \pi=$ $2.75 \mathrm{MHz} \mathrm{Oe}{ }^{-1}$ and hence, $g=1.9657$ which is slightly lower than the theoretically expected value of $g=2$ for ferromagnetic manganites. ${ }^{32}$ Belmeguenai et al. ${ }^{33}$ obtained $g=1.95$ in $\mathrm{La}_{0.7^{-}}$ $\mathrm{Sr}_{0.3} \mathrm{MnO}_{3}$ thin films using the microstrip FMR (MS-FMR) technique. In order to obtain an accurate value of $g$ factor, measurements need to be extended to several tens of GHz. As shown in the right inset of Fig. 6(b), $\Delta H$ increases non-linearly with frequency in the low frequency region but linearly in the high frequency regime. We have also extracted the values of $4 \pi M_{\mathrm{S}}$ and $H_{\mathrm{k}}$ from the fitting which are $1900 \pm 70$ Oe and $190 \pm$ 20 Oe, respectively.

In order to confirm whether the observed anomalous behaviour of the $\mathrm{MR}_{\mathrm{ac}}$ corresponds to FMR, we have fitted the field derivative of the power absorption $(\mathrm{d} P / \mathrm{d} H)$ by a combined function containing derivatives of symmetric and antisymmetric Lorentzian functions as ${ }^{34}$

$$
\begin{aligned}
\frac{\mathrm{d} P}{\mathrm{~d} H}= & P_{\mathrm{sym}} \frac{\frac{\Delta H}{2}\left(H_{\mathrm{dc}}-H_{\mathrm{res}}\right)}{\left[\left(H_{\mathrm{dc}}-H_{\mathrm{res}}\right)^{2}+\left(\frac{\Delta H}{2}\right)^{2}\right]^{2}} \\
& +P_{\text {asym }} \frac{\left(\frac{\Delta H}{2}\right)^{2}-\left(H_{\mathrm{dc}}-H_{\mathrm{res}}\right)^{2}}{\left[\left(H_{\mathrm{dc}}-H_{\mathrm{res}}\right)^{2}+\left(\frac{\Delta H}{2}\right)^{2}\right]^{2}}+P_{0}
\end{aligned}
$$

where, $P_{\text {sym }}$ and $P_{\text {asym }}$ are the coefficients of symmetric and antisymmetric Lorentzian derivatives, $H_{\text {res }}$ and $\Delta H$ are the resonance field and linewidth (FWHM), respectively for the $\mathrm{d} P /$ $\mathrm{d} H$ line shape and $P_{0}$ is the constant offset parameter. Fig. 7(a) displays the fitting of $\mathrm{d} P / \mathrm{d} H$ line shape with the eqn (8) for selected frequencies between $f=2$ and $10 \mathrm{GHz}$. The plot of frequency $(f) v s$. resonance field $\left(H_{\text {res }}\right)$ obtained from the fitting of $\mathrm{d} P / \mathrm{d} H$ line shapes are shown in main panel of Fig. 7(b). Fitting of the $f v s$. $H_{\text {res }}$ curve with eqn (4) yields $\gamma / 2 \pi=2.78 \mathrm{MHz}$ $\mathrm{Oe}^{-1}$ and hence, $g=1.987$ which is close to the value of $g$-factor extracted from the fitting of ac MR line shape.

The extracted the values of $4 \pi M_{\mathrm{S}}$ and $H_{\mathrm{k}}$ from the fitting of $\mathrm{d} P / \mathrm{d} H$ line shape are $2200 \pm 100$ Oe and $190 \pm 30$ Oe, respectively. It is to be noted that the value of $H_{\mathrm{k}}$ obtained from both magnetoimpedance and microwave absorption measurements are close to $H_{\mathrm{k}}=200 \pm 10$ Oe estimated from the fitting of $M(H)$ isotherm with the law of approach to saturation model which is expressed as, ${ }^{35}$

$$
M=M_{\mathrm{S}}\left(1-\frac{a}{H}-\frac{b}{H^{2}}\right)+\chi H
$$

where, the coefficient $a$ is related to micro-stress and the coefficient $b$ is connected to the first order magnetocrystalline anisotropy coefficient $K$ through the expression, $b=\frac{8}{105} \frac{K^{2}}{\mu_{0}^{2}}$. The anisotropy field $H_{\mathrm{k}}$ can be estimated from the relation: $H_{\mathrm{k}}{ }^{2}=$
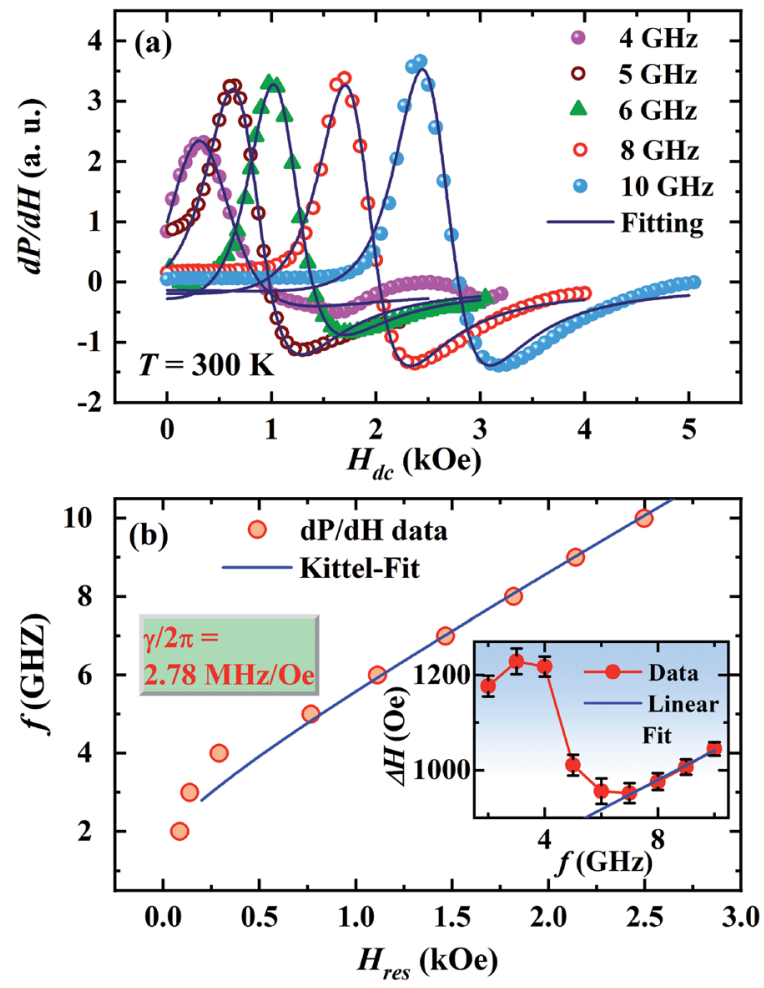

Fig. 7 (a) Fitting of $\mathrm{d} P / \mathrm{d} H$ line shapes at $T=300 \mathrm{~K}$ for different frequencies, (b) main panel shows the Kittel-fit to the plot of frequency (f) vs. resonance field $\left(H_{\text {res }}\right)$ extracted from $\mathrm{d} P / \mathrm{d} H$ data and the inset shows frequency dependence of $\Delta H$ with a linear fit for $f>6 \mathrm{GHz}$.

$2 K / \mu_{0} M_{\mathrm{S}}$. The frequency dependence of $\Delta H$ extracted from the fitting of $\mathrm{d} P / \mathrm{d} H$ curve is shown in the inset of Fig. 7(b) which clearly shows a nonlinear dependence below $6 \mathrm{GHz}$ but a linear dependence for higher frequencies.

Nonlinear $f$-dependence of $\Delta H$ was found in various magnetic nanostructures which was attributed to the presence of two relaxation processes: the intrinsic Gilbert's damping and the two-magnon scattering. ${ }^{36}$ The two-magnon scattering is an extrinsic damping mechanism which causes inhomogeneous broadening of $\Delta H$ and responsible for damping enhancement. We have fitted the $f$ dependence of $\Delta H$ above $6 \mathrm{GHz}$ with the following equation, ${ }^{37}$

$$
\Delta H=\Delta H_{0}+\frac{4 \pi \alpha}{\gamma} f
$$

where, $\Delta H_{0}$ accounts for the extrinsic damping contributions and $\alpha$ is the Gilbert's damping parameter. The value of $\alpha$ extracted from the fitting is $13.6 \times 10^{-3} \pm 0.8 \times 10^{-3}$ and it is higher than the value found in $\mathrm{La}_{0.7} \mathrm{Sr}_{0.3} \mathrm{MnO}_{3} / \mathrm{NGO}$ thin film $(\alpha$ $\left.=7.8 \times 10^{-4}\right)^{38}$ and Pt capped $\mathrm{La}_{0.7} \mathrm{Sr}_{0.3} \mathrm{MnO}_{3} / \mathrm{STO}$ thin film $(\alpha$ $\left.=5.93 \times 10^{-3}\right) \cdot{ }^{39} \mathrm{~A}$ possible reason for the large value of $\alpha$ observed could be due to polycrystalline nature of our sample. We need to investigate spin dynamics in epitaxial thin films by this magnetoimpedance technique in future.

In recent years, electrical detection of FMR using microstrip (MS) or co-planar waveguide (CPW) is gaining popularity to investigate spin dynamics in magnetic nanostructures. ${ }^{33,40}$ 
These techniques make use of a vector network analyzer (VNA) or a combination of MW signal generator, microwave diode and lock-in amplifier. The sample to be investigated is placed on the signal line of CPW or MS and the microwave magnetic field arising from the flow of MW current in the signal line induces spin precession in the sample. On the contrary, RF/WW current is directly injected into the sample in our method and our technique requires only an impedance analyzer to source MW current and record the impedance of the sample. Unlike a network analyzer, the rf impedance analyzer does not need a $50 \mathrm{ohm}$ impedance matching. Even though the maximum attainable frequency is limited to $3 \mathrm{GHz}$, there are ample opportunities to exploit this technique to other ferromagnetic systems.

\section{Summary}

In summary, we have studied the magnetic field dependence of electrical impedance in the room temperature ferromagnet $\mathrm{Pr}_{0.6} \mathrm{Sr}_{0.4} \mathrm{MnO}_{3}$ using an impedance analyzer by passing alternating current in the frequency range $1 \mathrm{MHz}$ to $3 \mathrm{GHz}$ directly through the sample. Our magnetoimpedance studies show signature of ferromagnetic resonance which was also confirmed by microwave absorption measurements for multiple frequencies $(f=2$ to $10 \mathrm{GHz})$ of microwave electromagnetic field using a commercial broadband ferromagnetic resonance spectrometer. Our line shape analysis indicates a large Gilbert's damping in this sample $\left(\alpha=13.6 \times 10^{-3}\right)$. Our results show that highfrequency magnetoimpedance can be used as a materials characterization tool. Ferromagnetic resonance do find applications in catalysis. ${ }^{41}$ Application of this magnetoimpedance technique to other conducting magnetic oxides may open up a new area of research direction in physical chemistry.

\section{Conflicts of interest}

There are no conflicts to declare.

\section{Acknowledgements}

R. M. acknowledges the Ministry of Education, Singapore for supporting this work (Grant numbers: R144-000-381-112 and R144-000-373-112).

\section{References}

1 C. N. R. Rao and A. K. Raychaudhuri, "Colossal Magnetoresistance, Charge Ordering and Related Properties of Manganese Oxides", ed. C. N. R. Rao and B. Raveau, World Scientific, Singapore, 1998 pp. 1-42.

2 Y. Tokura and Y. Tomioka, J. Magn. Magn. Mater., 1999, 200, 1.

3 T. Aoyama, K. Yamauchi, A. Iyama, S. Picozzi, K. Shimizu and T. Kimura, Nat. Commun., 2014, 5, 4927; T. Arima, J. Phys. Soc. Jpn., 2011, 80, 052011.

4 M. Aparnadevi and R. Mahendiran, AIP Adv., 2013, 3, 012114.
5 H. Qin, J. Hu, B. Li, Y. Hao, J. Chen and M. Jiang, J. Magn. Magn. Mater., 2008, 320, 2770; J. Hu, H. Qin, B. Li, Y. Wang and Y. Zhang, J. Magn. Magn. Mater., 2011, 323, 1185.

6 A. Rebello, V. Naik and R. Mahendiran, J. Appl. Phys., 2009, 106, 073905.

7 H. Chen, J. Hu, J. Chen, Y. Wang and Z. Wang, J. Appl. Phys., 2002, 91, 10003.

8 B. Singh, Phys. Chem. Chem. Phys., 2016, 18, 12947.

9 M. Dominguez, S. M. Bhagat, S. E. Lofland, J. S. Ramachandran, G. C. Xiong, H. L. Ju, T. Venkatesan and R. L. Greene, Europhys. Lett., 1995, 32, 349.

10 V. V. Srinivasu, S. E. Lofland, S. M. Bhagat, K. Ghosh and S. Tyagi, J. Appl. Phys., 1999, 86, 1067.

11 C. Ritter, P. G. Radaelli, M. R. Lees, J. Baratt, G. Balakrishnan and D. M. Paul, J. Solid State Chem., 1996, 127, 276.

12 S. Rößler, H. S. Nair, U. K. Rößler, C. M. N. Kumar, S. Elizabeth and S. Wirth, Phys. Rev. B: Condens. Matter Mater. Phys., 2011, 84, 184422.

13 D. V. Maheswar Repaka, T. S. Tripathi, M. Aparnadevi and R. Mahendiran, J. Appl. Phys., 2012, 112, 123915.

14 S. Rosler, S. Harikrishnan, C. M. N. Kumar, H. L. Bhat, S. Elizabeth, U. K. Rosler, F. Steglich and S. Wirth, J. Supercond. Novel Magn., 2009, 22, 205.

15 R. Thaljaoui, W. Boujelben, M. Pękała, J. Szydłowska and A. Cheikhrouhou, J. Alloys Compd., 2012, 526, 98; R. Thaljaoui, W. Boujelben, M. Pekała, J. Szydłowska and A. Cheikhrouhou, EPJ Web Conf., 2012, 29, 00050.

16 A. I. Shames, E. Rozenberg, W. H. McCarroll, M. Greenblatt and G. Gorodetsky, Phys. Rev. B: Condens. Matter Mater. Phys., 2001, 64, 172401.

17 G. Srinivasan and R. Suryanarayanan, J. Magn. Magn. Mater., 2002, 242, 686.

18 J. Yang, X. Rong, D. Suter and Y. P. Sun, Phys. Chem. Chem. Phys., 2011, 13, 16343.

19 A. Chanda and R. Mahendiran, J. Appl. Phys., 2018, 124, 043902.

20 L. Kraus, Sens. Actuators, A, 2003, 106, 187.

21 L. V. Panina, K. Mohri and T. Uchiyama, Phys. A, 1997, 241, 429; L. V. Panina, K. Mohri, K. Bushida and M. Noda, J. Appl. Phys., 1994, 76, 6198.

22 M. Ipatov, V. Zhukova, A. Zhuklov and J. Gonzalez, Sci. Rep., 2016, 6, 36180.

23 A. M. H. de Andrade, M. A. Corrêa, A. D. C. Viegas, F. Bohn and R. L. Sommer, J. Appl. Phys., 2014, 115, 103908.

24 C. Kittel, Phys. Rev., 1948, 73, 155.

25 M. R. Britel, D. Ménard, L. G. Melo, P. Ciureanu, A. Yelon, R. W. Cochrane, M. Rouabhi and B. Cornut, Appl. Phys. Lett., 2000, 77, 2737.

26 J. M. Barandiarán, A. García-Arribas and D. de Cos, J. Appl. Phys., 2006, 99, 103904.

27 D. de Cos, G. Alvarez, A. Garia-Arribas, H. Montiel, J. M. Barandiaran, R. Zamorono and R. Valenzuela, Sens. Actuators, A, 2008, 142, 485.

28 Y.-Y. Song, S. Kalarickal and C. E. Patton, J. Appl. Phys., 2003, 94, 5103.

29 M. Harder, Z. X. Cao, Y. S. Gui, X. L. Fan and C.-M. Hu, Phys. Rev. B: Condens. Matter Mater. Phys., 2011, 84, 054423. 
30 T. Nan, S. Emori, C. T. Boone, X. Wang, T. M. Oxholm, J. G. Jones, B. M. Howe, G. J. Brown and N. X. Sun, Phys. Rev. B: Condens. Matter Mater. Phys., 2015, 91, 214416.

31 N. Mecking, Y. S. Gui and C.-M. Hu, Phys. Rev. B: Condens. Matter Mater. Phys., 2007, 76, 224430.

32 V. A. Ivanshin, J. Deisenhofer, H.-A. Krug von Nidda, A. Loidl, A. A. Mukhin, A. M. Balbashov and M. V. Eremin, Phys. Rev. B: Condens. Matter Mater. Phys., 2000, 61, 6213.

33 M. Belmeguenai, S. Mercone, C. Adamo, L. Méchin, C. Fur, P. Monod, P. Moch and D. G. Schlom, Phys. Rev. B: Condens. Matter Mater. Phys., 2010, 81, 054410.

34 P. Dürrenfeld, F. Gerhard, J. Chico, R. K. Dumas, M. Ranjbar, A. Bergman, L. Bergqvist, A. Delin, C. Gould, L. W. Molenkamp and J. Akerman, Phys. Rev. B: Condens. Matter Mater. Phys., 2015, 92, 214424.

35 B. D. Cullity, and C. D. Graham, Introduction to magnetic materials. John Wiley \& Sons, 2011, p. 325.
36 K. Lenz, H. Wende, W. Kuch and K. Baberschke, Phys. Rev. B: Condens. Matter Mater. Phys., 2006, 73, 144424.

37 S. S. Kalarickal, P. Krivosik, M. Wu, C. E. Patton, M. L. Schneider, P. Kabos, T. J. Silva and J. P. Nibarger, J. Appl. Phys., 2006, 99, 093909.

38 Q. Qin, S. He, W. Song, P. Yang, Q. Wu, Y. P. Feng and J. Chen, Appl. Phys. Lett., 2017, 110, 112401.

39 G. Y. Luo, M. Belmeguenai, Y. Roussigné, C. R. Chang, J. G. Lin and S. M. Chérif, AIP Adv., 2015, 5, 097148.

40 H. K. Lee, I. Barsukov, A. G. Swartz, B. Kim, L. Yang, H. Y. Hwang and I. N. Krivorotov, AIP Adv., 2016, 6, 055212. 41 E. G. Derouane, A. J. Simoens and J. C. Vedrine, Chem. Phys. Lett., 1977, 52, 549; E. G. Derouane, A. J. Simoens, C. Colin, G. A. Martin, J. A. Dalmon and J. C. Vedrine, J. Catal., 1978, 52, 50 . 